\title{
A model for nonlinear viscoelastic torsional response of an elastomeric bushing
}

\author{
S. B. Lee and A. Wineman, Ann Arbor, Michigan \\ (Received August 27, 1997; revised January 14, 1998)
}

\begin{abstract}
Summary. An elastomeric bushing is a device used in automotive suspension systems to cushion the loads transmitted from the wheel to the frame of the vehicle. A bushing is essentially an elastomeric hollow cylinder which is bonded to a solid metal shaft at its inner surface and a metal sleeve at its outer surface. The shaft is connected to the suspension and the sleeve is connected to the frame. The elastomeric cylinder provides the cushion when it deforms due to relative motion between the shaft and the sleeve. The relation between the force or moment applied to the shaft or sleeve and the relative displacements or rotations is nonlinear and exhibits features of viscoelasticity.

A force(moment)-displacement(rotation) relation for elastomeric bushings is important for multibody dynamics numerical simulations. A boundary value problem for the bushing response leads to a relation which requires extensive computation time to implement and is hence unsuitable. In a separate study, a force(moment)-displacement(rotation) relation for single mode response has been proposed which can be used in multi-body dynamics simulations. The relation is expressed in terms of a force (moment) relaxation function for the bushing, and a method for its determination by experiments on bushings has been presented. The applicability of this relation for torsional mode bushing response is evaluated in the present work.

A boundary value problem is formulated for torsional mode bushing response. Numerical solutions of the boundary value problem represent the exact bushing response and act as numerically generated experimental data. The proposed moment-rotation relation is constructed using this data. Numerical solutions of the boundary value problem also allow for comparison between the exact moment-rotation behavior and that predicted by the proposed model. It is shown that the method for determining the bushing relaxation function and the predictions of the proposed moment-rotation relation are in very good agreement with the exact results.
\end{abstract}

\section{Introduction}

Elastomeric bushings are structural elements which are used in suspension systems of automobiles and other vehicles. A bushing is, in effect, a hollow cylinder of elastomeric material contained between an outer steel cylindrical sleeve and an inner steel cylindrical rod. The steel sleeve and rod are connected to components of the automotive suspension system and are used to transfer forces and moments from the wheel through the elastomeric material to the chassis. The elastomeric material is used to reduce shocks and vibrations in the connection. Because they are connected to different parts of the suspension system, the sleeve and rod undergo relative axial displacements along and rotations about their common centerline, as well as along and about axes perpendicular to the centerline. It is this relative motion that stresses the elastomeric material and allows for the transmission of forces and moments through the bushing.

In analyzing suspension systems that contain bushings, engineers have made use of multibody dynamics analysis, a tool of computer-aided engineering used in many engineering and 
manufacturing industries, and especially in the automotive industry. Experience using this tool has led engineers at Ford Motor Company to conclude that it is essential to choose the correct bushing model in order to obtain accurate predictions of the dynamic behavior of the suspension system and, in particular, to obtain accurate predictions of the dynamic loads that act on the suspension system components which are supported by bushings (Ledesma et al. [1]). In these multi-body dynamics codes, the bushings are typically modeled by using a system of force-displacement relations (used here in the generalized sense to also mean a relation between moment and rotation). Thus, determining the correct force-displacement relation for a bushing becomes an important task. This task, however, is made difficult by the presence of the elastomeric material in the bushing. This material is viscoelastic and nonlinear; hence, it causes the relation between a force or moment and its corresponding displacement to be nonlinear and time dependent, as well as causing coupling between the different modes of response. Experimental data from single mode tests carried out at the Center for Automotive Structural Durability Simulation at the University of Michigan indicate that a bushing force has a nonlinear dependence on the corresponding displacement and that the nonlinearity is manifested under normal operating conditions. The data also indicate that bushings exhibit characteristics of viscoelastic response such as force relaxation and hysteresis under sinusoidal oscillations. This viscoelasticity cannot be neglected because it is a mechanism for energy dissipation and, thus, analyzing its effect is essential for accurate calculations.

There are two basic approaches which can be used to determine the required force-displacement relation for a bushing. The first, a standard approach used in applied mechanics, combines a three-dimensional constitutive equation for the bushing elastomeric material with the equations of equilibrium and strain-displacement equations to define an appropriate boundary value problem describing that mode of response. Because of the nonlinearity of response, this procedure does not generally result in an explicit mathematical relation between force and displacement. If a force or displacement is specified, the other quantity is determined by solving the boundary value problem. The advantage of this approach is that, in concept, it can be used to calculate the force-deformation relation for any desired bushing design. One disadvantage of this approach is that the development of a constitutive equation for nonlinear viscoelastic response is in itself a very formidable task involving an extensive experimental program. Very few examples of such constitutive equations appear in the literature. A second disadvantage is that the force-displacement relation is defined implicitly as the solution to a boundary value problem. Using this model requires repeated numerical solutions of this boundary value problem. This is computationally intensive and, hence, is undesirable for incorporation in multi-body dynamics simulations.

The second approach is to attempt to determine this force-displacement relation directly from experiments. The advantage of this approach is that it produces an explicit force-displacement relation which is convenient for later use in multi-body dynamics analysis. On the other hand, a disadvantage is that the process must be repeated for each new bushing design. Another disadvantage is that it does not allow for the examination of the response of the elastomeric material at a local level.

Very few studies of the development of force-displacement relations for bushings have been found in the open literature. In the studies that do exist, the first approach mentioned above is used exclusively. Adkins and Gent [2] developed force-displacement relations for radial, axial, torsional, and conical modes of deformation of bonded cylindrical bushings. Their results were obtained using the linear theory of elasticity and thus do not account for nonlinear, time dependent effects, or coupling between modes. Morman, Kao, and Nagtegaal [3] modeled the elastomeric bushing material as a nonlinear viscoelastic solid. They assumed 
an appropriate constitutive equation and presented a finite element method for analyzing the response for small amplitude oscillations superposed on large equilibrium deformations. Although their method is important for studying many aspects of bushing response, it does not account for transient response and is thus of limited use in a multi-body dynamics code. If other studies have been performed, they are proprietary and not available in the open literature.

In [4], Wineman, VanDyke, and Shi used the second approach and suggested a force-displacement relation for single mode bushing response which combines nonlinear dependence on displacement with the time dependence of viscoelasticity. It is expressed in terms of a force relaxation function, a bushing property which is to be determined directly by experiment. The force relaxation function represents the force which arises when the bushing is subjected to a step displacement history. Although this displacement history cannot be realized in an actual experimental program, it can be approached by an appropriate sequence of displacement histories. A method was presented for determining the force relaxation function by extrapolating the measured force responses obtained in an experimental program in which a bushing is subjected to this sequence of displacement histories. The method was implemented using data obtained in radial mode response. This force-displacement relation has been incorporated in a multi-body dynamics code (see Ledesma et al. [1]).

The purpose of the present work is to assess the applicability of the approach introduced in [4] for the development of a moment-rotation relation for torsional mode response. This is done by formulating a boundary value problem for torsional mode bushing response as would be done in the first approach discussed above. Simulations carried out with the equations for this boundary value problem represent the exact bushing response. One set of simulations produces numerically generated 'experimental' data which are used to determine the moment relaxation function property in the proposed moment-rotation relation. A second set of simulations is used to compare the predictions of the moment-rotation relation and the mathematical model for the exact response.

While it would be desirable to carry out this assessment using general nonlinear viscoelastic properties of the elastomeric bushing material, such a study is beyond the scope of this work. In the present work, numerical comparisons are made using a single specific constitutive model. This constitutive equation for the nonlinear viscoelastic response of the elastomeric bushing material is presented in Section 2. The moment-rotation relation for the bushing introduced in [4] and the method for measuring its moment relaxation function are outlined in Section 3. The formulation of the mathematical model for torsional mode response of a bushing is presented in Section 4. This mode of response is selected so as to take advantage of simplifications arising from axial symmetry and material incompressibility. Because of the nonlinearities in the exact bushing model, an explicit relation between moment and rotation is not possible. Instead, determination of the exact moment or rotation history, when the other is specified, requires the solution of a system of nonlinear integral equations. Solutions of this system for several moment and rotation histories are presented in Section 5. In Section 6, the extrapolation method for determining the moment relaxation property is carried out using numerically generated 'experimental' data obtained from the exact mathematical model. It is shown that there is very good agreement between the moment relaxation function obtained by the extrapolation method and the exact one. Solutions of the proposed moment-rotation relation and of the mathematical model for exact bushing response are compared in Section 7. The results show that the proposed moment-rotation relation can provide a good approximation to the exact one. Section 8 contains final comments. 


\section{Constitutive equation for elastomeric bushing material}

The current time is denoted by $t$, and $s$ denotes an ealier time, so that $-\infty<s \leq t$. Let $\mathbf{X}$ denote the position of a material particle in the reference configuration, which is also taken as the undeformed configuration. The position of the material particle at time $s$ is $\mathrm{x}(s)$. The motion of a body is then decribed by a relation of the form

$\mathbf{x}(s)=\chi(\mathbf{X}, s), \quad-\infty<s \leq t$.

The deformation gradient at the particle at time $s$ is denoted by $\mathbf{F}(s)$. Its components with respect to a Cartesian coordinate system are given by

$\mathbf{F}(s)=\left\{\frac{\partial x_{i}(s)}{\partial X_{j}}\right\}$

The motion is assumed to be smoothly invertible, that is, $\operatorname{det} \mathbf{F}(s) \neq 0$, so that

$\mathbf{X}=\chi^{-1}(\mathbf{x}(\mathbf{s}), \mathbf{s})$.

The left Cauchy-Green strain tensor is defined by $\mathbf{B}(t)=\mathbf{F}(t)[\mathbf{F}(t)]^{\mathrm{T}}$. With respect to a Cartesian coordinate system, its invariants $\mathbf{I}_{1}$ and $\mathbf{I}_{2}$ are given by

$\mathbf{I}_{1}=B_{i i}, \quad \mathbf{I}_{2}=\frac{1}{2}\left\{B_{i i} B_{j j}-B_{i j} B_{i j}\right\}$.

The assumption of incompressibility requires that $\operatorname{det} \mathbf{F}(t)=1$. For notational convenience, $\mathbf{B}=\mathbf{B}(t)$.

Let the relation in Eq. (2.3) be evaluated at the current time $t$, and then be combined with that in Eq. (2.1). The result is a description of the motion at time $s$ relative to that at time $t$,

$\mathbf{x}(s)=\hat{\chi}(\mathbf{x}(t) ; s, t)$.

The relative deformation gradient is then denoted by $\mathrm{F}_{t}(s)$, and its components with respect to Cartesian coordinates are given by

$\mathbf{F}_{t}(s)=\left\{\frac{\partial x_{i}(s)}{\partial x_{j}(t)}\right\}$

This can be expressed in terms of the deformation gradient defined in Eq. (2.2) by

$\mathbf{F}_{t}(s)=\mathbf{F}(s) \mathbf{F}^{-1}(t)$.

The right relative Cauchy-Green tensor is defined by $\mathbf{C}_{t}(s)=\left[\mathbf{F}_{t}(s)\right]^{\mathbf{T}} \mathbf{F}_{t}(s)$, and the material time derivative of $\mathbf{C}_{t}(s)$ is defined by

$\dot{\mathbf{C}}_{t}(s)=\frac{d \mathbf{C}_{t}(s)}{d s}$

A three-dimensional constitutive theory for the isothermal response of an incompressible isotropic viscoelastic material has been developed by Coleman and Noll [5]. This theory utilizes the notion of fading memory to develop an approximation to the exact constitutive equation. When the deformation is carried out sufficiently slowly, in a sense that can be made mathematically precise, then the first order approximation expresses the stress as a single time integral whose integrand depends on appropriate strain tensors. 
Lianis and co-workers [6]-[11] carried out an extensive experimental program to determine the specific form of the single integral constitutive equation for styrene-butadiene rubber under isothermal conditions. They arrived at a final form of the constitutive equation by a process described in [10] as "involving trial and error, cross plotting and curve fitting over a wide range of uniaxial and biaxial relaxation data." By experimental verification, they showed that their constitutive equation produces accurate results for other deformation histories.

The constitutive equation developed by Lianis and co-workers has the form

$$
\begin{aligned}
\sigma(t)= & -\hat{p}(t) \mathbf{I}+\left(a+\frac{b}{\left(I_{1}-2\right)^{2}}+I_{1}\left[c+d\left(I_{2}-3\right)\right]\right) \mathbf{B}-\left[c+d\left(I_{2}-3\right)\right] \mathbf{B}^{2} \\
& +2 \int_{-\infty}^{t}\left[P_{0}(t-s)+\left(I_{2}-3\right) Q_{0}(t-s)\right] \dot{\mathbf{C}}_{t}(s) d s \\
& +\int_{-\infty}^{t}\left(P_{1}(t-s)+\frac{Q_{1}(t-s)}{\left(I_{1}-2\right)^{2}}\right)\left[\mathbf{B} \dot{\mathbf{C}}_{t}(s)+\dot{\mathbf{C}}_{t}(s) \mathbf{B}\right] d s
\end{aligned}
$$

where $\hat{p}$ is a scalar which arises from the constraint of incompressibility. $P_{0}(t), Q_{0}(t), P_{1}(t)$, $Q_{1}(t), a, b, c$, and $d$ are material properties and are determined from the experimental data. The material constants $(a, b, c, d)$ and the values of the material property functions represented by $\left(P_{0}(t), Q_{0}(t), P_{1}(t), Q_{1}(t)\right)$ are given at the set of times shown in Table 1 [10]. When time $t$ is different from the values listed in Table $1, P_{0}(t), Q_{0}(t), P_{1}(t)$ and $Q_{1}(t)$ are evaluated by using linear extrapolation and interpolation. Linear extrapolation is used to obtain the values from $t=0$ to $t=1 \mathrm{sec}$. and linear interpolation is used above $t=1 \mathrm{sec}$.

\section{Nonlinear viscoelastic torsional moment-rotation relation}

As discussed in the Introduction, we consider a moment-rotation relation for the bushing which combines nonlinear dependence on rotation and time effects of viscoelasticity. The simplest relation which meets these conditions appears to be that introduced by Pipkin and Rogers [12] for the nonlinear viscoelastic response of polymers. In the current context, it has the form

$M(t)=R(\Delta(t), 0)+\int_{0}^{t} \frac{\partial R(\Delta(s), t-s)}{\partial(t-s)} d s$,

in which $M(t)$ and $\Delta(t)$ denote the torsional moment and angle of rotation at time $t$, respectively. By applying integration by parts to Eq. (3.1), the Pipkin-Rogers model is rewritten as follows:

$M(t)=R(\Delta(0), t)+\int_{0}^{t} \frac{\partial R(\Delta(s), t-s)}{\partial \Delta(s)} \frac{d \Delta(s)}{d s} d s$.

$R(\Delta, t)$ is a property of the bushing and represents the moment at time $t$ due to a step rotation of amount $\Delta$ applied at time zero. That is, $R(\Delta, t)$ can be interpreted as the rotation dependent moment relaxation function. It is reasonable to assume that $R\left(0^{-}, t\right)=0$ and that $R(\Delta, t)$ is a monotonically decreasing function of $t$.

Ideally, $R(\Delta, t)$ can be determined by subjecting the bushing to a deformation in which the outer shell undergoes a step rotation with respect to the inner rod. This ideal process can- 
not be realized due to the inertia of the testing apparatus. A method which can be used to determine $R(\Delta, t)$ has been developed and presented in [4] and is outlined here.

Consider a rotation history which increases at a constant rate to angle $\Delta_{i}$ in time interval $T_{j}{ }^{*}$, and is then held fixed. This history is described by

$$
\begin{array}{rlrl}
\Delta(t) & =\frac{\Delta_{i}}{T_{j}^{*}} t, & 0 \leq t \leq T_{j}^{*}, \\
& =\Delta_{i}, \quad T_{j}^{*} \leq t,
\end{array}
$$

where $T_{j}{ }^{*}$ is defined as the rise time. Let this rotation history be denoted by $\Delta\left(\Delta_{i}, T_{j}{ }^{*}, t\right)$ and be called a ramp rotation control test.

The moment history corresponding to this ramp rotation history can be expected to increase monotonically until time $T_{j}{ }^{*}$, and then relax for times $t>T_{j}{ }^{*}$, when the rotation is held constant. As $T_{j}{ }^{*}$ approaches zero, the ramp rotation control test approaches the step rotation control test. Moreover, as $T_{j}^{*}$ approaches zero, the moment response can be expected to approach the rotation dependent moment relaxation function $R\left(\Delta_{i}, t\right)$. In order to show this, let Eq. (3.3) be substituted into Eq. (3.2), and consider any time $t \geq T_{j}{ }^{*}$. Since $R(\Delta(0), t)=R(0, t)=0$ and $d \Delta(s) / d s=0, s \geq T_{j}{ }^{*}$,

$M(t)=\int_{0}^{T_{j}^{*}} \frac{\partial R(\Delta(s), t-s)}{\partial \Delta(s)} \frac{d \Delta(s)}{d s} d s$.

This moment will be denoted by $\tilde{R}\left(\Delta_{i}, t, T_{j}^{*}\right)$.

In Eq. (3.4), let the integration variable be changed from $s$ to $\Delta=\Delta_{i} s / T_{j}^{*}$. Also, let $t=T_{j}^{*}+\hat{t}$, where $\hat{t}$ denotes a fixed time measured from $T_{j}^{*}$, when the rotation is held constant. Then, Eq. (3.4) becomes

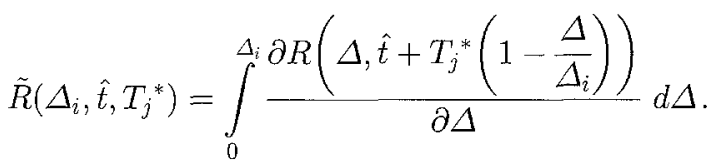

As $T_{j}^{*}$ approaches zero, Eq. (3.5) becomes

$\lim _{T_{j}^{*} \rightarrow 0} \tilde{R}\left(\Delta_{i}, \hat{t}, T_{j}^{*}\right)=\int_{0}^{\Delta_{i}} \frac{\partial R(\Delta, \hat{t})}{\partial \Delta} d \Delta=R\left(\Delta_{i}, \hat{t}\right)$.

Let $\Delta_{i}, i=1,2,3, \ldots, n_{\Delta}$ be the set of angles of rotation and let $T_{j}^{*}, j=1,2,3, \ldots, n_{T^{*}}$ be the set of rise times at which the ramp rotation history described in Eq. (3.3) is carried out. Consider a fixed value of rotation $\Delta_{i}$ and let the rise time $T_{j}{ }^{*}$ decrease as $j$ increases, that is, $T_{j+1}^{*}<T_{j}^{*}$. A set of such histories is shown in Fig. la. Because of experimental limitations, the limit $T_{j}^{*} \rightarrow 0$ cannot be reached. This difficulty is approached as follows.

Let $\hat{R}_{\exp }\left(\Delta_{i}, T_{j}^{*}, t\right)$ denote the experimentally determined moment history response at time $t \geq T_{j}^{*}$. When the slope of ramp rotation history $\Delta\left(\Delta_{i}, T_{j}^{*}, t\right)$ changes from positive to zero at the rise time $T_{j}{ }^{*}$, the slope of the moment-time response changes from positive to negative at time $T_{j}{ }^{*}$.

Let $\hat{t}$ be a fixed time interval measured from this break in the slope at time $T_{j}^{*}$, that is, $t=\hat{t}+T_{j}{ }^{*}$. For the rise times $T_{j}^{*}, j=1,2,3, \ldots, n_{T^{*}}, \hat{R}_{\exp }\left(\Delta_{i}, T_{j}{ }^{*}, T_{j}{ }^{*}+\hat{t}\right)$ denotes a set of data points associated with time $\hat{t}$ and rotation $\Delta_{i}$. Figure $1 \mathrm{~b}$ shows plots of the experimentally determined moment history $\hat{R}_{\exp }\left(\Delta_{i}, T_{j}{ }^{*}, t\right)$ versus $t$, at a fixed value of $\hat{t}$, for several rise 


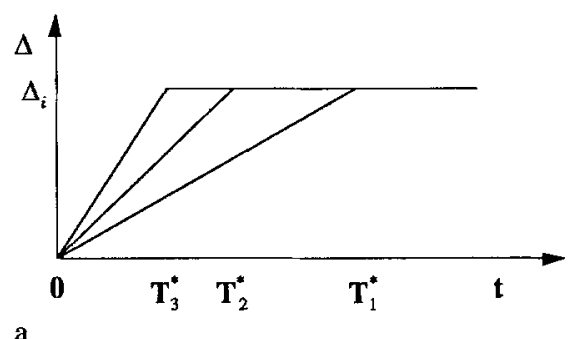

a

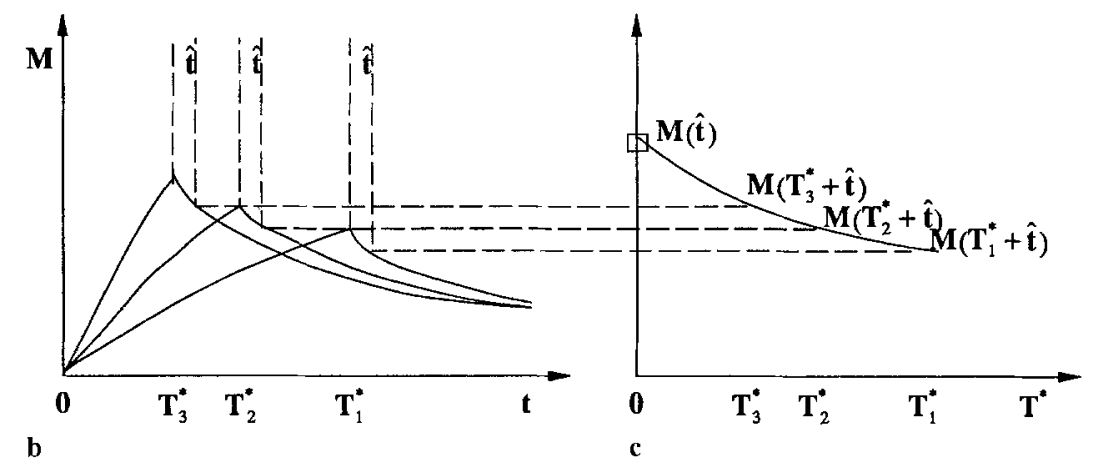

Fig. 1. Ramp rotation control tests a Rotation history (input), b Moment (output), c Moment extrapolation

times $T_{j}^{*}$. Figure $1 \mathrm{c}$ shows a plot of $\hat{R}_{\exp }\left(\Delta_{i}, T^{*}, T^{*}+\hat{t}\right)$ versus $T^{*}$, indicating the values corresponding to $\hat{t}$ and the rise times of Fig. $1 \mathrm{~b}$.

Let $\hat{R}_{\mathrm{fit}}\left(\Delta_{i}, \hat{t}, T^{*}\right)$ denote a function of $T^{*}$ which is fit to this set of data points, so that

$\hat{R}_{f i t}\left(\Delta_{i}, \hat{t}, T_{j}^{*}\right)=\hat{R}_{\exp }\left(\Delta_{i}, T_{j}^{*}, T_{j}^{*}+\hat{t}\right), \quad T_{j}^{*}, j=1,2,3, \ldots, n_{T^{*}}$.

$\hat{R}_{f i t}\left(\Delta_{i}, \hat{t}, T^{*}\right)$ is used to extrapolate the data for $T_{j}^{*}, j=1,2,3, \ldots, n_{T^{*}}$ to $T^{*}=0$. In particular, it is assumed that

$$
\begin{aligned}
\lim _{T^{* \rightarrow \rightarrow 0}} \hat{R}_{f i t}\left\{\Delta_{i}, \hat{t}, T^{*}\right) & =\hat{R}_{f i t}\left(\Delta_{i}, \hat{t}, 0\right) \\
& \approx \lim _{T^{*} \rightarrow 0} \hat{R}_{\exp }\left(\Delta_{i}, T_{j}^{*}, T_{j}^{*}+\hat{t}\right) \\
& =R\left(\Delta_{i}, \hat{t}\right) .
\end{aligned}
$$

A choice for $\hat{R}_{f i t}\left(\Delta_{i}, \hat{t}, T^{*}\right)$ was developed by assuming that in Eq. (3.4) $\partial R(\Delta, x) / \partial \Delta=$ $g_{0}+g_{1} \exp (-x / \tau)$ for some set of constants $g_{0}, g_{1}$, and $\tau$. This leads to the relation

$\hat{R}_{f i t}\left(\Delta_{i}, \hat{t}, T_{j}^{*}\right)=C_{1}+C_{2}\left(\frac{1-e^{-\left(T^{*} / \tau\right)}}{\left(T^{*} / \tau\right)}\right)$.

$C_{1}, C_{2}$, and $\tau$ are constants which are found by minimizing

$\sum_{j=1}^{n_{T^{*}}}\left(\left(C_{1}+C_{2}\left(\frac{1-e^{-\left(T_{j}^{*} / \tau\right)}}{\left(T_{j}^{*} / \tau\right)}\right)\right)-\hat{R}_{\exp }\left(\Delta_{i}, T_{j}^{*}, T_{j}^{*}+\hat{t}\right)\right)^{2}$. 
By substituting Eq. (3.9) into Eq. (3.8) and using the result that $\lim _{T^{*} \rightarrow 0}\left[\left(1-e^{-\left(T^{*} / \tau\right)}\right) /\left(T^{*} / \tau\right)\right]$ $=1$, it is found that

$R\left(\Delta_{i}, \hat{t}\right)=C_{1}+C_{2}$.

The process indicated in Eqs. (3.10) and (3.11) can be carried out on any specified set of angles of rotation $\Delta_{i}$ and times $\hat{t}$. The result provides the values $R\left(\Delta_{i}, \hat{t}\right)$ on this set.

\section{Response of elastomeric bushing material}

The bushing is treated as a long hollow cylinder of elastomeric material bonded to a long rigid rod at its inner surface and a long rigid sleeve at its outer surface. End effects and inertia are ignored and the system is axially symmetric. In this study, only axially symmetric deformations are considered.

The reference configuration, which is the undeformed configuration, is referred to cylindrical coordinates $R, \theta$, and $Z$. The current configuration is referred to cylindrical coordinates denoted by $r, \theta$, and $z$. The bushing is at rest in its reference configuration for times $t<0$. For times $t \geq 0$, the inner rod is subjected to a torsional moment $M(t)$, and undergoes a rotation with respect to the outer sleeve. During the deformation, the cylindrical surface at radius $R$ undergoes an angle of rotation, which is denoted by $g(R, t)$. After taking incompressibility into account, the motion of the cylinder is then described by

$r(R, t)=R$,

$\theta(R, t)=\Theta+g(R, t)$

$z(R, t)=Z$.

Since the outer sleeve is held fixed, $g\left(R_{o}, t\right)=0$. The inner rod undergoes rotation $g\left(R_{i}, t\right)$.

The components of the deformation gradient and the relative stretch tensor with respect to cylindrical coordinates are given by

$\mathbf{F}(R, t)=\left[\begin{array}{ccc}1 & 0 & 0 \\ R \frac{\partial g(R, t)}{\partial R} & 1 & 0 \\ 0 & 0 & 1\end{array}\right]=\left[\begin{array}{ccc}1 & 0 & 0 \\ h(R, t) & 1 & 0 \\ 0 & 0 & 1\end{array}\right]$

and

$\mathbf{F}_{t}(R, s)=\mathbf{F}(R, s) \mathbf{F}^{-1}(R, t)=\left[\begin{array}{ccc}1 & 0 & 0 \\ \{h(R, s)-h(R, t)\} & 1 & 0 \\ 0 & 0 & 1\end{array}\right]$,

where $h(R, t)=R \partial g(R, t) / \partial R$ denotes shear in the circumferential direction.

It is convenient to describe the motion of the cylinder in three time intervals: (i) at rest with $g(R, s)=0$ for $s<0^{-}$, (ii) an instantaneous deformation from $s=0^{-}$to $s=0^{+}$with $g\left(R, 0^{-}\right)=0$ and $g\left(R, 0^{+}\right) \neq 0$, and (iii) a continuous motion for $s>0^{+}$. The quantities which appear in Eq. (2.9), $\mathbf{B}$ and $\mathbf{B}^{2}$, their invariants $\mathbf{I}_{1}$ and $\mathbf{I}_{2}$, and $\mathbf{C}_{t}(s)$, can be found from Eqs. (4.2) and (4.3). By substituting these into Eq. (2.9) and then accounting for the three separate time intervals of the motion, expressions for the normal and shear stresses are obtained. The calculation is lengthy and is omitted for the sake of brevity. Details can be found in [13]. 
The relation between shear stress $\sigma_{r \theta}(R, t)$ and shear strain history $h(R, s)$ is given by

$$
\begin{aligned}
\sigma_{r \theta}(R, t)= & \left(a+\frac{b}{\left[1+h^{2}(R, t)\right]^{2}}+\left[3+h^{2}(R, t)\right]\left[c+d h^{2}(R, t)\right]\right) h(R, t) \\
& -\left[c+d h^{2}(R, t)\right]\left[2+h^{2}(R, t)\right] h(R, t)+2 h\left(R, 0^{+}\right)\left[P_{0}(t)+h^{2}(R, t) Q_{0}(t)\right] \\
& +2 \int_{0^{+}}^{t}\left(P_{0}(t-s) \frac{\partial h(R, s)}{\partial s}\right) d s+2 h^{2}(R, t) \int_{0^{+}}^{t}\left(Q_{0}(t-s) \frac{\partial h(R, s)}{\partial s}\right) d s \\
& +h\left(R, 0^{+}\right)\left(P_{1}(t)+\frac{Q_{1}(t)}{\left[1+h^{2}(R, t)\right]^{2}}\right)\left[2+h\left(R, 0^{+}\right) h(R, t)-h^{2}(R, t)\right] \\
& +2 h(R, t) \int_{0^{+}}^{t}\left(P_{1}(t-s) h(R, s) \frac{\partial h(R, s)}{\partial s}\right) d s \\
& +\left[2-h^{2}(R, t)\right] \int_{0^{+}}^{t}\left(P_{1}(t-s) \frac{\partial h(R, s)}{\partial s}\right) d s \\
& +\frac{2 h(R, t)}{\left[1+h^{2}(R, t)\right]^{2}} \int_{0^{+}}^{t}\left(Q_{1}(t-s) h(R, s) \frac{\partial h(R, s)}{\partial s}\right) d s \\
& +\frac{2-h^{2}(R, t)}{\left[1+h^{2}(R, t)\right]^{2}} \int_{0^{+}}^{t}\left(Q_{1}(t-s) \frac{\partial h(R, s)}{\partial s}\right) d s .
\end{aligned}
$$

Note that $\sigma_{r \theta}(R, t)$ is odd in the shear strain history. It can be shown that the normal stresses are expressed in terms of $\hat{p}(R, t)$ and the shear strain history. They will not be used in the remainder of this paper and are omitted for the purpose of brevity.

As mentioned above, the inertia of the bushing is ignored. After accounting for axial symmetry, the equations of motion reduce to

$$
\begin{aligned}
& \frac{\partial \sigma_{r r}}{\partial r}+\frac{\sigma_{r r}-\sigma_{\theta \theta}}{r}=0, \\
& \frac{\partial \sigma_{r \theta}}{\partial r}+\frac{2}{r} \sigma_{r \theta}=0 .
\end{aligned}
$$

Equations (4.4) and (4.6) can be used to determine $h(R, s)$. In the remainder of this Section, the emphasis will be on these equations for $h(R, s)$.

Note that Eq. (4.6) can be integrated to give

$\sigma_{r \theta}(R, t)=\frac{M(t)}{2 \pi R^{2} L}$,

where $M(t)$ is the torsional moment on the inner rod.

Let $G$ denote the shear modulus which is defined by

$$
\lim _{h\left(R, 0^{+}\right) \rightarrow 0} \frac{\sigma_{r \theta}\left(R, 0^{+}\right)}{h\left(R, 0^{+}\right)}=G .
$$

It follows from Eq. (4.4) that

$$
G=a+b+c+2\left\{P_{0}\left(0^{+}\right)+P_{1}\left(0^{+}\right)+Q_{1}\left(0^{+}\right)\right\} .
$$


The values for $a, b, c$ and $P_{0}\left(0^{+}\right), P_{1}\left(0^{+}\right), Q_{1}\left(0^{+}\right)$in Table 1 give $G=107.95(p s i)$.

Let the non-dimensional radius $\hat{R}$ be defined by $\hat{R}=R / R_{i}$. Then, $\hat{R}_{i}=1$ and $\hat{R}_{o}=R_{o} / R_{i}$ denotes the ratio of the outer radius to the inner radius. Since rotation $g(R, t)$ is dimensionless, $\hat{g}(\hat{R}, t)=g(R, t)$, and the non-dimensional shear is

$\hat{h}(\hat{R}, s)=\hat{R} \frac{\partial \hat{g}(\hat{R}, s)}{\partial \hat{R}}$

Let the non-dimensional moment $\hat{M}(t)$ be defined by

$\hat{M}(t)=\frac{M(t)}{2 \pi R_{i}^{2} L G}$.

The shear stress and shear strains in Eq. (4.4) are regarded as functions of the non-dimensional radius $\hat{R}$. Then, using Eqs. (4.9) and (4.11), Eq. (4.7) becomes

$\sigma_{r \theta}(\hat{R}, t)=G \frac{\hat{M}(t)}{\hat{R}^{2}}$

Combining Eq. (4.12) and Eq. (4.4) gives a relation among $\hat{M}(t), \hat{R}$, and $(\hat{h}(\hat{R}, s)$, $0 \leq s \leq t)$. The non-dimensional rotation at the inner radius $\hat{g}(1, t)$ is calculated from

$\hat{g}(1, t)=-\int_{1}^{\hat{R}_{o}} \frac{\hat{h}(\hat{R}, t)}{\hat{R}} d \hat{R}$.

The relation between the torsional moment $\hat{M}(t)$ and the rotation at the inner radius $\hat{g}(1, t)$ is defined by the system of Eqs. (4.4), (4.12), and (4.13). $\hat{M}(t)$ appears explicitly in Eq. (4.12). When $\hat{M}(t)$ is specified, Eqs. (4.4) and (4.12) become a nonlinear Volterra integral equation for the shear strain history $(\hat{h}(\hat{R}, s), 0 \leq s \leq t) . \hat{h}(\hat{R}, t)$ can be determined at each radius $\hat{R}$, and then $\hat{g}(1, t)$ can be found by using Eq. (4.13). Conversely, when the rotation history at the inner radius $(\hat{g}(1, s), 0 \leq s \leq t)$ is specified, the corresponding moment history $\hat{M}(t)$ must be determined by an iteration process. Let $\hat{M}^{(n)}(t)$ denote the $n^{\text {th }}$ iteration for the moment, and let $\hat{g}^{(n)}(1, t)$ denote the rotation at the inner radius which is determined by the process outlined above. Moment iteration $\hat{M}^{(n+1)}(t)$ is chosen so that $\hat{g}^{(n+1)}(1, t)$ converges to the specified rotation $\hat{g}(1, t)$. Because of the complexity of the system of Eqs. (4.4), (4.12), and (4.13), this process requires much more computation time. In summary, the moment-rotation relation consists of a computationally intensive procedure.

\section{Exact bushing response}

The bushing model just presented is now used to determine the moment responses to several specified rotation histories. These represent possible experimental control conditions, and the responses represent numerically generated 'experimental' results. Two rotation histories are considered, the step rotation history

$\hat{g}(1, t)=\hat{g}_{0}, \quad t \geq 0$,

and the ramp rotation history

$$
\begin{aligned}
\hat{g}(1, t) & =\frac{\hat{g}_{0}}{T^{*}} t, \quad 0 \leq t \leq T^{*}, \\
& =\hat{g}_{0}, \quad t \geq T^{*} .
\end{aligned}
$$


Table 1. Material functions for SBR at $0^{\circ} \mathrm{C}$

\begin{tabular}{lllll}
\hline Time $(\mathrm{sec})$ & $P_{0}(t) \mathrm{psi}$ & $Q_{0}(t) \mathrm{psi}$ & $P_{1}(t) \mathrm{psi}$ & $Q_{1}(t) \mathrm{psi}$ \\
\hline 1 & 4.30 & -0.575 & 4.95 & 4.05 \\
2 & 4.10 & -0.575 & 3.675 & 3.05 \\
4 & 3.735 & -0.535 & 2.75 & 2.25 \\
6 & 3.45 & -0.510 & 2.40 & 1.80 \\
10 & 3.05 & -0.470 & 1.95 & 1.30 \\
15 & 2.70 & -0.415 & 1.625 & 1.05 \\
20 & 2.50 & -0.375 & 1.425 & 0.875 \\
30 & 2.15 & -0.310 & 1.150 & 0.725 \\
40 & 1.92 & -0.275 & 0.95 & 0.61 \\
50 & 1.75 & -0.235 & 0.825 & 0.55 \\
60 & 1.60 & -0.215 & 0.75 & 0.5 \\
120 & 1.05 & -0.125 & 0.425 & 0.31 \\
180 & 0.75 & -0.08 & 0.25 & 0.225 \\
360 & 0.20 & -0.025 & 0.125 & 0.1 \\
540 & 0.05 & 0 & 0.05 & 0.05 \\
720 & 0 & 0 & 0 & 0 \\
\hline
\end{tabular}

${ }^{*} P_{0}(0)=4.5 ; Q_{0}(0)=-0.575 ; P_{1}(0)=6.225 ; Q_{1}(0)=5.05$.

$*_{\mathrm{a}}=43.7 \mathrm{psi} ; \mathrm{b}=16.7 \mathrm{psi} ; \mathrm{c}=16 \mathrm{psi} ; \mathrm{d}=-1.25 \mathrm{psi}$.

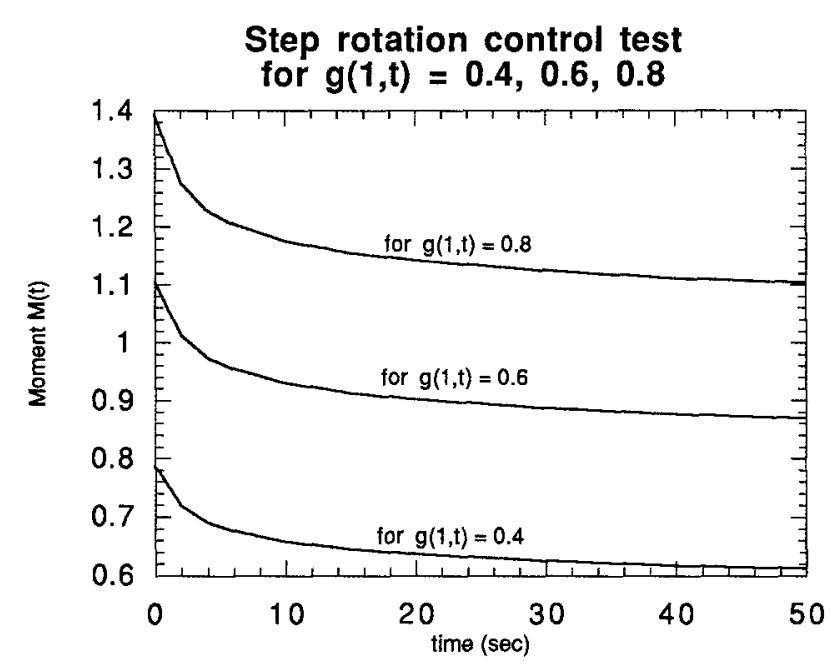

Fig. 2. Step rotation control test for $\hat{g}(1, t)=0.4,0.6,0.8$

Results for step rotation histories $\left(\hat{g}_{0}=0.4,0.6,0.8\right)$ are shown in Fig. 2. Note that the moments have almost reached their fully relaxed values when $t=50 \mathrm{sec}$. This is consistent with the behavior of the material relaxation properties in Table 1 which are close to their long time equilibrium values when $t=50 \mathrm{sec}$. Note that a significant amount of moment relaxation occurs.

Results for ramp rotation histories $\left(\hat{g}_{0}=0.8, T^{*}=2,4,6,8 \mathrm{sec}\right.$.) are shown in Fig. 3a. Note that $\hat{M}\left(T^{*}\right)$ increases as $T^{*}$ decreases and appears to approach a limiting value as $T^{*} \rightarrow 0$. Also note that $\hat{M}(50)$ is independent of $T^{*}$, illustrating the property of fading memory. 

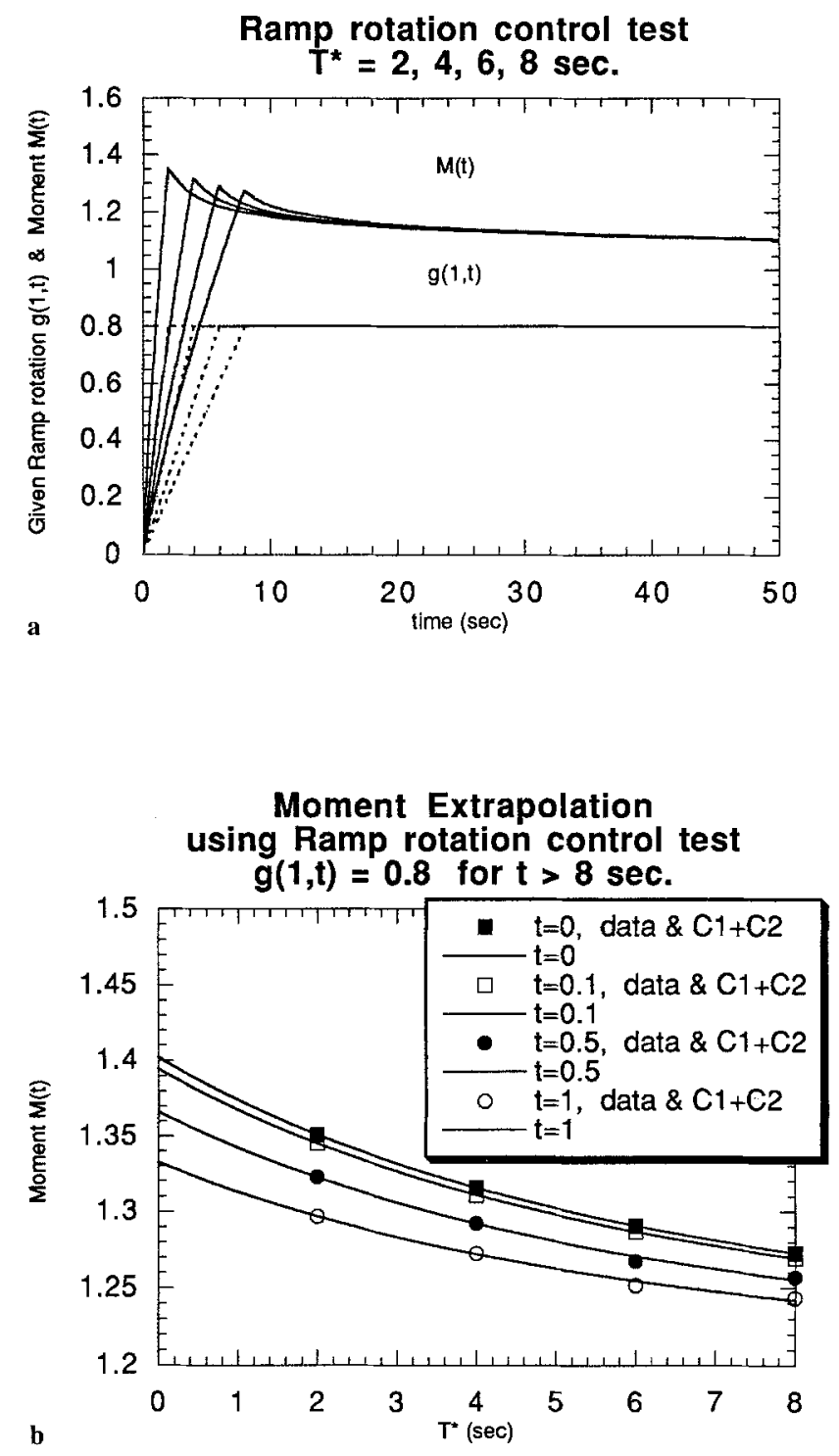

Fig. 3

Shear strain histories at the inner surfaces $\hat{h}(1, t)$ were computed, but details are omitted here for the purpose of brevity. It is worth mentioning results for step rotation control tests. Although $\hat{g}(1, t)$ is constant, the shear strain $\hat{h}(1, t)$ decreases in time. Thus, according to Eq. (4.13), the shear strain distribution varies with time in order to keep $\hat{g}(1, t)$ constant. For details, see [13].

Rotation histories were computed for step moment histories and ramp moment histories, the latter of the form in Eq. (5.2). Figures showing these results are omitted here for the purpose of brevity, and may be found in [13]. Results for step moment histories show that there is a significant amount of creep. They also show the nonlinearity of response, in the sense that when the moments are scaled by some factor, the rotations are not. Results for ramp moment histories show that $\hat{g}\left(1, T^{*}\right)$ appears to approach a limiting value as $T^{*} \rightarrow 0$. They also show that $\hat{g}(1,50)$ is independent of $T^{*}$, which illustrates the property of fading memory. 


\section{Moment relaxation function $R(d, t)$ for $d=0.8$}
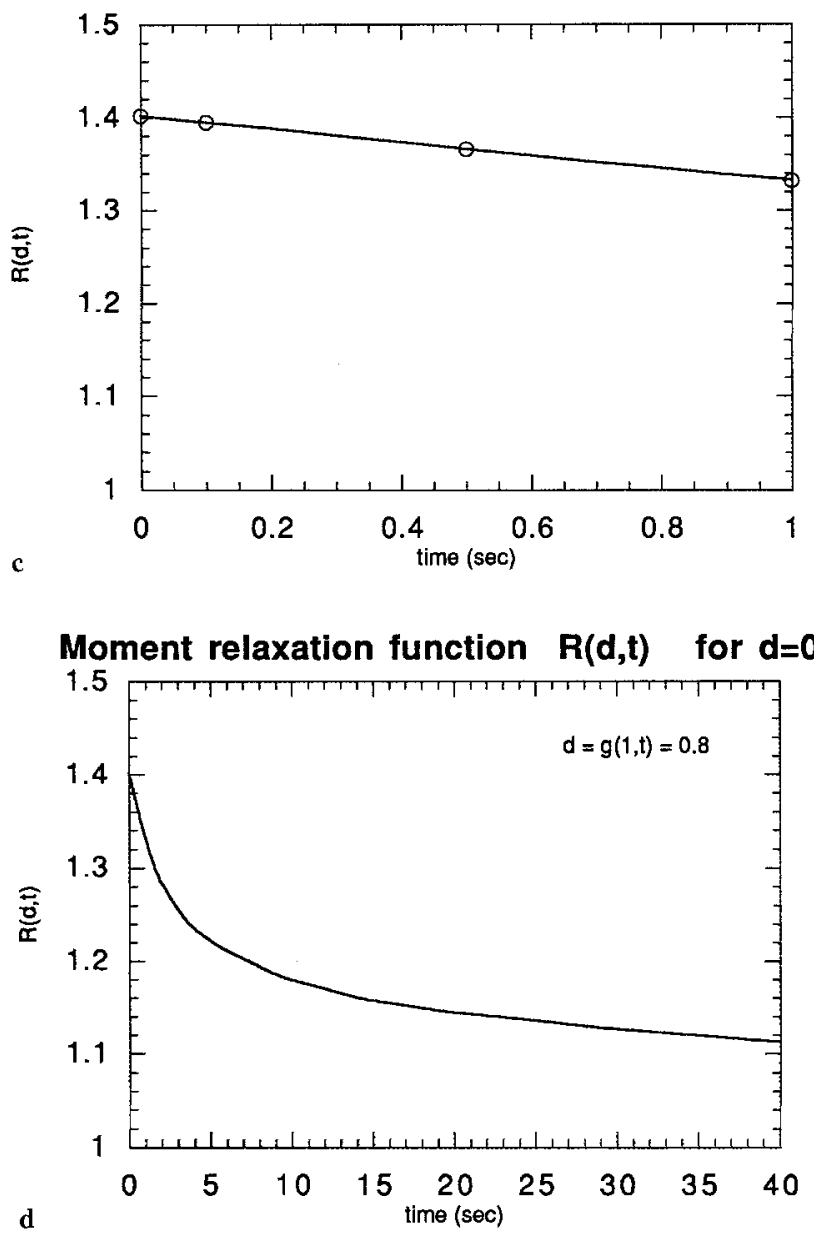

Fig. 3. a Ramp rotation control test b Moment extrapolation c Moment relaxation function for $0 \leq t \leq 1 \mathrm{sec}$. d Moment relaxation function

\section{Explicit moment-rotation relation}

The moment-rotation relation given by the system of Eqs. (4.4), (4.12), and (4.13) is an exact one, restricted only by the modeling assumptions. If this system of equations were to be used in practical applications, computationally intensive numerical methods would be required in order to determine one history when the other is specified. In this Section, we consider a relation in which the torsional moment is expressed explicitly in terms of the rotation history, namely, the Pipkin-Rogers model presented in Section 3. This relation provides an approximation to that given by the exact system, but requires less computation time. It also accounts for the nonlinear viscoelastic response of the bushing illustrated in Section 5.

For present purpose, we use the form of the Pipkin-Rogers model in Eq. (3.2) for bushing response in torsional motion,

$\hat{M}(t)=R(\hat{g}(1,0), \hat{t})+\int_{0^{+}}^{t} \frac{\partial R(\hat{g}(1, s), t-s)}{\partial \hat{g}(1, s)} \frac{d \hat{g}(1, s)}{d s} d s$,

where $R(\hat{g}, t)$ is the rotation dependent moment relaxation function. We now explore this model as an approximation to the exact moment-rotation response for torsional motion. 
Table 2. The cases which were carried out for the extrapolation procedure

\begin{tabular}{lllll}
\hline$\hat{g}_{0}$ & $T_{1}{ }^{*}(\mathrm{sec})$ & $T_{2}{ }^{*}(\mathrm{sec})$ & $T_{3}{ }^{*}(\mathrm{sec})$ & $T_{4}{ }^{*}(\mathrm{sec})$ \\
\hline 0.2 & 8 & 6 & 4 & 2 \\
0.4 & 8 & 6 & 4 & 2 \\
0.6 & 8 & 6 & 4 & 2 \\
0.8 & 8 & 6 & 4 & 2 \\
1.0 & 8 & 6 & 4 & 2 \\
\hline
\end{tabular}

Table 3. The values of $\left(\hat{t}, \tau, C_{1}+C_{2}\right)$ obtained from the extrapolation process for $\hat{g}=0.8$

\begin{tabular}{lll}
\hline$\hat{t}$ (second) & $\tau$ (second) & $C_{1}+C_{2}$ \\
\hline 0 & 3.4148 & 1.4023 \\
0.1 & 3.4148 & 1.3948 \\
0.5 & 3.4148 & 1.3665 \\
1.0 & 3.4148 & 1.3326 \\
\hline
\end{tabular}

\section{I Determination of moment relaxation function from the exact model}

The moment relaxation function $R(\hat{g}, t)$ was determined by the process outlined in Section 3 . Ramp rotation control tests were simulated numerically using the exact model defined by Eqs. (4.4), (4.12), and (4.13). The moment histories were treated as numerically generated experimental data which are to be used for finding $R(\hat{g}, t)$.

The ramp rotation histories at the inner radius $\hat{g}(1, t)$ have the form

$$
\begin{aligned}
\hat{g}(1, t) & =\frac{\hat{g}_{0}}{T_{j}^{*}} t, \quad 0 \leq t \leq T_{j}^{*}, \\
& =\hat{g}_{o}, \quad T_{j}^{*} \leq t .
\end{aligned}
$$

The extrapolation procedure outlined in Section 3 was carried out for each value of $\hat{g}_{0}$ and $T_{j}^{*}$ listed in Table 2 . Note that the rotation at the inner radius $\hat{g}(1, t)$ and the non-dimensional torsional moment $\hat{M}(t)$ correspond respectively to $\Delta\left(\Delta_{i}, T_{j}^{*}, t\right)$ and $\hat{R}_{\exp }\left(\Delta_{i}, T_{j}^{*}, t\right)$ in Section 3.

Results are discussed for $\hat{g}_{0}=0.8$. Similar comments apply to $\hat{g}_{0}=0.2,0.4,0.6$. The calculated non-dimensional moment data $\hat{R}_{\exp }\left(\hat{g}_{0}, T^{*}, T^{*}+\hat{t}\right)$ vs. $T^{*}$ are represented by the symbols at $T^{*}=2,4,6,8 \mathrm{sec}$. in Fig. $3 \mathrm{~b}$. The extrapolation functions $\hat{R}_{f i t}\left(\hat{g}_{0}, \hat{t}, T^{*}\right)$ defined in Eq. (3.9) were fit to these data for several choices of $\hat{t}$ and are also plotted in Fig. 3b. The values of parameters in these fits are shown in Table 3. The intersections of these functions with the vertical axis gives the limits in Eqs. (3.8) and (3.11), that is, $\hat{R}_{f i t}\left(\hat{g}_{0}, \hat{t}, 0\right)=R\left(\hat{g}_{0}, \hat{t}\right)$ $=C_{1}+C_{2}$. The values of $R\left(\hat{g}_{o}, \hat{t}\right)$ for $\hat{t}=0,0.1,0.5,1 \mathrm{sec}$. are indicated by the circles in Fig. $3 \mathrm{c}$, which shows the moment relaxation function for $\hat{g}_{0}=0.8$ on the time interval $0 \leq t \leq 1 \mathrm{sec}$. This process was carried out for a set of times $\hat{t}, 0 \leq \hat{t} \leq 40 \mathrm{sec}$, and the moment relaxation function on this time interval is shown in Fig. 3d.

These results can be used to evaluate the extrapolation process. This is done by comparing the moment relaxation function determined by the extrapolation process in Section 3, 
Moment relaxation function $\mathbf{R}(\mathbf{g}, \mathbf{t})$
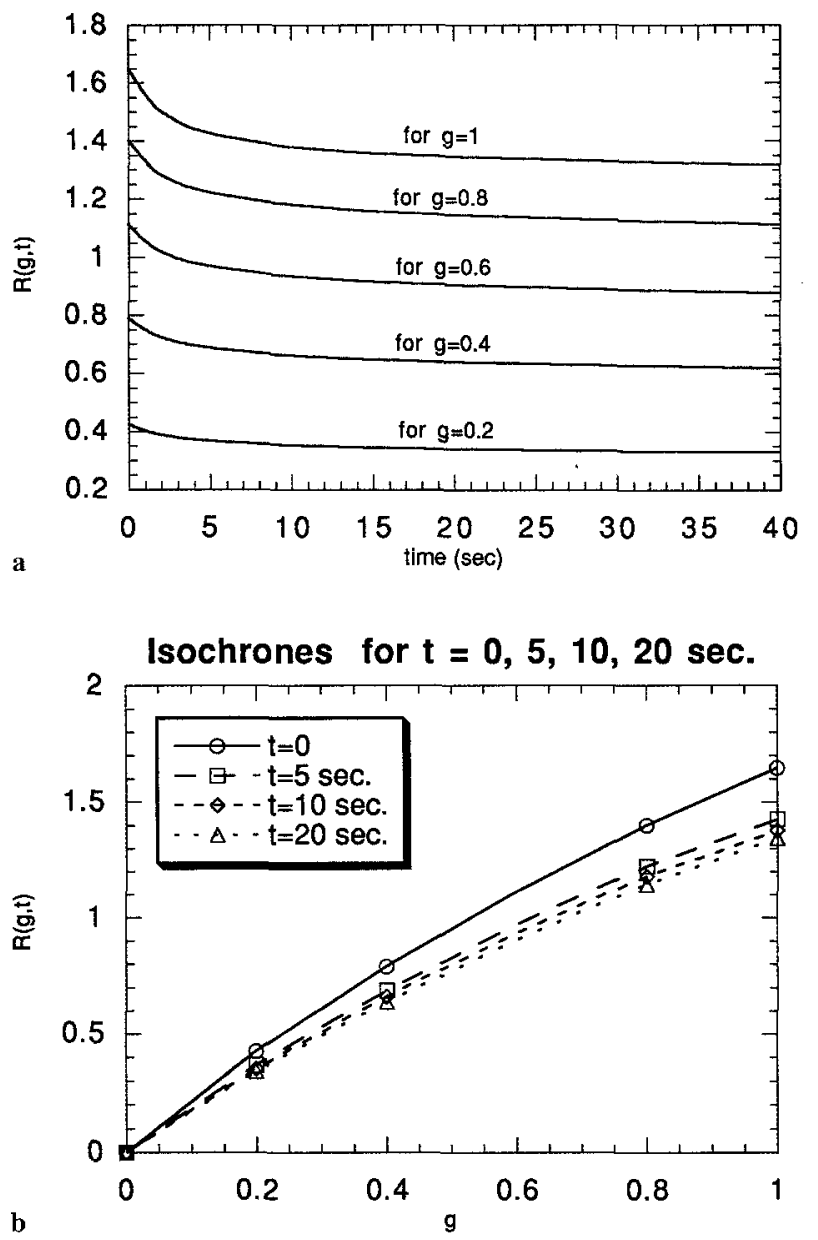

Fig. 4. a Moment relaxation function $\mathbf{b}$ Isochrones for $t=0,5,10,20 \mathrm{sec}$.

$\hat{R}_{f i t}\left(\hat{g}_{0}, \hat{t}, 0\right)$, with the moment relaxation function determined using the exact model in Section $5, R\left(\hat{g}_{0}, \hat{t}\right)$,

$E_{\text {extrap }}(\hat{t})=\left|\frac{\hat{R}_{f i t}\left(\hat{g}_{0}, \hat{t}, 0\right)-R\left(\hat{g}_{0}, \hat{t}\right)}{R\left(\hat{g}_{0}, \hat{t}\right)}\right|$.

At $\hat{t}=0$, for $\hat{g}_{0}=0.2,0.4,0.6, E_{\text {extrap }} \approx 0.006$ and $E_{\text {extrap }} \approx 0.007$ for $\hat{g}_{0}=0.8$. Thus, at $\hat{t}=0$, this extrapolation has a relative error below $1 \%$. The largest errors occurred for $\hat{t}<10 \mathrm{sec}$, and were less than $1 \%$. For $10 \leq \hat{t} \leq 40 \mathrm{sec}$, the error was much less than $1 \%$. Therefore, the extrapolation process can be regarded as reliable.

\subsection{Mathematical representation of $R(\hat{g}, t)$ for the Pipkin-Rogers model}

The moment relaxation functions for $\hat{g}_{0}=0.2,0.4,0.6,0.8,1$ are shown together in Fig. 4 a. Note that the curves in Fig. 4 a were produced by plotting the values of $\hat{R}_{f i t}\left(\hat{g}_{0}, \hat{t}, 0\right)$ and letting the computer graphics program produce a smooth curve. Isochrones of $R(\hat{g}, t)$ at $t=0$, $5,10,20$ sec. are shown in Fig. 4 b. It was found that the isochrones could be fit by a fifth degree polynomial in the rotation $\hat{g}$. Because the shear stress $\sigma_{r \theta}$ is odd in the shear strain, 
the polynomial contains only odd powers of $\hat{g}$. Thus, $R(\hat{g}, t)$ was represented in the form

$R(\hat{g}, t)=\hat{g} G_{1}(t)+\hat{g}^{3} G_{3}(t)+\hat{g}^{5} G_{5}(t)$.

The moment relaxation functions shown in Fig. 4 a represent a data set which can be used for finding $\left(G_{1}\left(t_{i}\right), G_{3}\left(t_{i}\right), G_{5}\left(t_{i}\right)\right)$. Let $\hat{g}^{(a)}$ be a value of a step rotation. Recall that $\hat{R}_{f i t}\left(\hat{g}^{(a)}\right.$, $\left.t_{i}, 0\right)$ denotes the value of the extrapolated moment relaxation corresponding to $\hat{g}^{(a)}$ and $t_{i}$. Then, $\left(G_{1}\left(t_{i}\right), G_{3}\left(t_{i}\right), G_{5}\left(t_{i}\right)\right)$ were determined to minimize the least squares error

$E\left(t_{i}\right)=\sum_{a=1}^{5}\left(R\left(\hat{g}^{(a)}, t_{i}\right)-\hat{R}_{f i t}\left(\hat{g}^{(a)}, t_{i}, 0\right)\right)^{2}$

where, by (6.4),

$R\left(\hat{g}^{(a)}, t_{i}\right)=\hat{g}^{(a)} G_{1}\left(t_{i}\right)+\left(\hat{g}^{(a)}\right)^{3} G_{3}\left(t_{i}\right)+\left(\hat{g}^{(a)}\right)^{5} G_{5}\left(t_{i}\right)$.

A description of this least squares minimization process is omitted for brevity, details can be found in [14]. The coefficients $\left(G_{1}\left(t_{i}\right), G_{3}\left(t_{i}\right), G_{5}\left(t_{i}\right)\right)$ produced by this process are obtained at a set of times $t_{i}$ for $0 \leq t_{i} \leq 40 \mathrm{sec}$. and are shown in Fig. $5 \mathrm{a}$. Note that the plots were produced by specifying data points and letting the computer graphics program produce a smooth curve.

It was found that this data can be represented by sums of exponential functions,

$\tilde{G}_{i}(t)=C_{i 1}+C_{i 2} e^{-t / \tau_{i 2}}+C_{i 3} e^{-t / \tau_{i 3}}+C_{i 4} e^{-t / \tau_{i 4}}$,

where $i=1,3,5$. The parameters $\left(C_{i \alpha}, \tau_{i \beta}, \alpha=1,2,3,4, \beta=2,3,4\right)$ were found by using the nonlinear least squares method [15], [16] and are shown in Table 4.

Let the relative error of the fit of $\tilde{G}_{i}(t)$ to the data represented by $G_{i}(t)$ be defined by

$E_{i}=\frac{\sqrt{\sum_{\gamma=1}^{401}\left(\tilde{G}_{i}\left(t_{\gamma}\right)-G_{i}\left(t_{\gamma}\right)\right)^{2}}}{\sqrt{\sum_{\gamma=1}^{401}\left(G_{i}\left(t_{\gamma}\right)\right)^{2}}}$,

where $i=1,3,5$. The relative errors are $E_{1}=0.0013, E_{3}=0.0018, E_{5}=0.0134$. Therefore, the data $\left(G_{i}\left(t_{\gamma}\right), i=1,3,5,0 \leq t_{\gamma} \leq 40 \mathrm{sec}\right.$. $)$ can be approximated by $\left(\tilde{G}_{i}(t), i=1,3,5\right)$ in Eq (6.7) with a relative error below $2 \%$. The plots of the data points and of the expressions in Eq. (6.7) are nearly indistinguishable in Fig. 5 a. The difference between the values for $G_{1}(t)$ in Fig. $5 \mathrm{a}$ and the values determined from Eq. (6.7) is shown in Fig. 5 b.

The complete form of the Pipkin-Rogers model for the torsional motion obtained by combining Eqs. (6.1), (6.4), and (6.7) is as follows:

$\hat{M}(t)=R(\hat{g}(1,0), t)+\int_{0^{+}}^{t} \frac{\partial R(\hat{g}(1, s), t-s)}{\partial \hat{g}(1, s)} \frac{d \hat{g}(1, s)}{d s} d s$,

where

$$
\begin{aligned}
R(\hat{g}(1, t), t)= & \left(C_{11}+C_{12} e^{-t / \tau_{12}}+C_{13} e^{-t / \tau_{13}}\right) \hat{g}(1, t) \\
& +\left(C_{31}+C_{32} e^{-t / \tau_{32}}+C_{33} e^{-t / \tau_{33}}\right)(\hat{g}(1, t))^{3} \\
& +\left(C_{51}+C_{52} e^{-t / \tau_{52}}+C_{53} e^{-t / \tau_{53}}+C_{54} e^{-t / \tau_{54}}\right)(\hat{g}(1, t))^{5},
\end{aligned}
$$

where $\left(C_{i \alpha}, \tau_{i \beta}, \alpha=1,2,3,4, \beta=2,3,4\right)$ are in Table 4 . 
Table 4. Parameters related to moment relaxation function

\begin{tabular}{|c|c|c|c|c|c|c|c|}
\hline$i$ & $C_{i 1}$ & $C_{i 2}$ & $C_{i 3}$ & $C_{i 4}$ & $T_{i 2}$ & $\tau_{i 3}$ & $\tau_{i 4}$ \\
\hline 1 & 1.6114 & 0.2448 & 0.2400 & 0 & 15.6895 & 2.0877 & Not applicable \\
\hline 3 & -0.4951 & -0.1020 & -0.1387 & 0 & 12.3654 & 2.8285 & Not applicable \\
\hline 5 & 0.1839 & 0.1238 & -0.0170 & 0.0046 & 2.4729 & 0.5733 & 0.0573 \\
\hline
\end{tabular}

\section{Coefficients of Moment relaxation function}
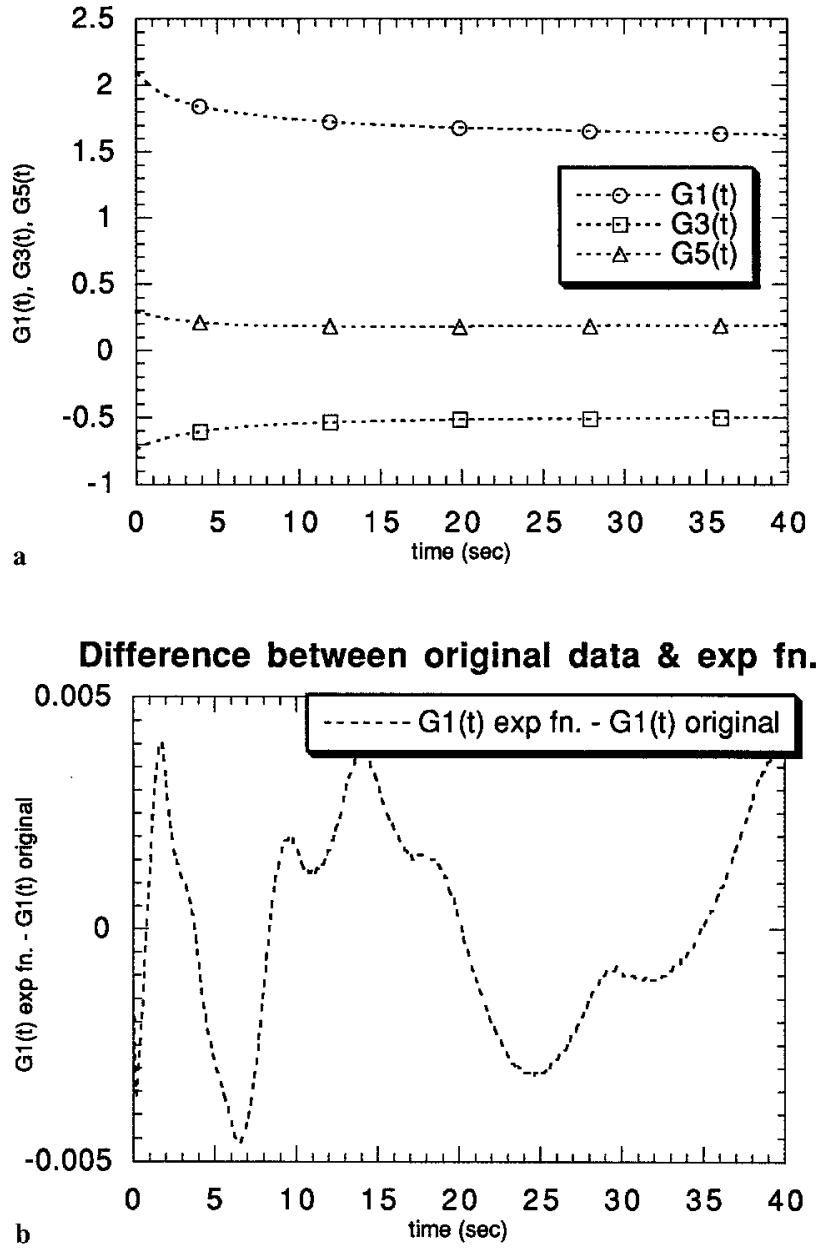

Fig. 5. a Time dependent coefficients: $G_{1}(t), G_{3}(t), G_{5}(t)$, b Difference between $G_{1}(t)$ in Fig. 5 a and from Eq. (6.7)

\section{Predictive quality of the Pipkin and Rogers model}

The Pipkin-Rogers model in Section 6 was introduced as an approximation to the exact model defined by the system of Eqs. (4.4), (4.12), and (4.13). The purpose of this Section is to discuss the accuracy of the Pipkin-Rogers model as an approximation to the exact model. Both the exact model and the Pipkin-Rogers model were used to calculate the moment responses to specified rotation histories. For evaluation purposes, the relative error $E$ is defined by

$E=\frac{\| \text { (Pipkin and Rogers output })-(\text { Exact output }) \|}{\| \text { (Exact output }) \|}$. 
The particular rotation histories used are the constant rate rotation and recovery histories defined as follows:

$$
\begin{array}{rlrl}
\hat{g}(1, t) & =\frac{0.8}{\tilde{t}} t, & & 0 \leq t \leq \tilde{t} \\
& =-\frac{0.8}{\tilde{t}}(t-2 \tilde{t}), & \tilde{t} \leq t \leq 2 \tilde{t} \\
& =0, & & 2 \tilde{t} \leq t \leq 40 \mathrm{sec},
\end{array}
$$

where $\tilde{t}$ is the rise time.

Fig. 6 a shows the rotation history in Eq. (7.2) for $\tilde{t}=10 \mathrm{sec}$. and the moment response history computed using the exact model. The moment response history for the same rotation history was also computed using the Pipkin-Rogers model. The difference between the moment responses for the exact and Pipkin-Rogers models is very small and is shown in Fig. 6 b. The relative error is 0.0288 . Calculations were also carried out with $\tilde{t}=5 \mathrm{sec}$, and the relative error was 0.0303 (see [13]). For those two cases, the relative errors are about $3 \%$ and the Pipkin-Rogers model appears to provide satisfactory agreement with the exact model.
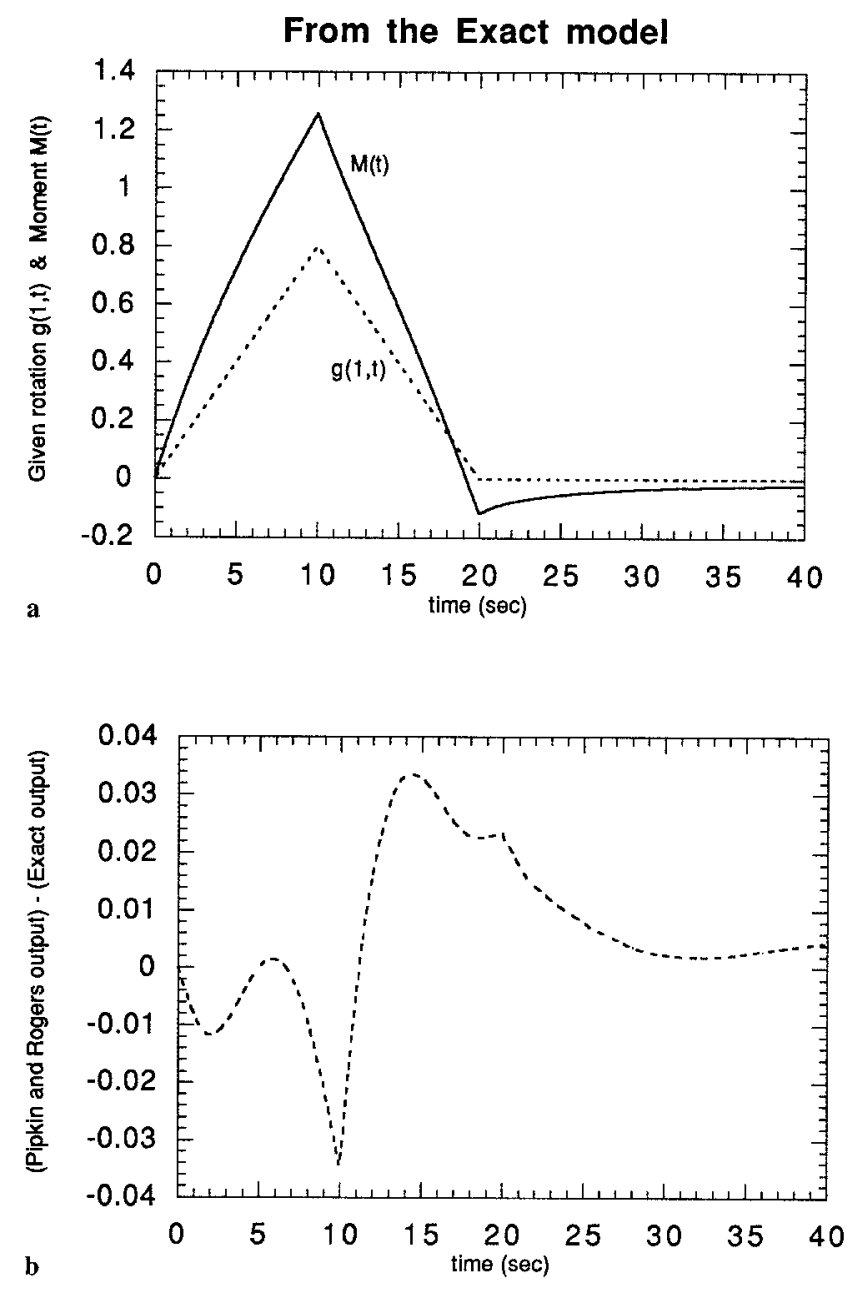

Fig. 6. a Rotation control test using the exact model, b Difference in $\hat{M}(t)$ between exact and Pipkin-Rogers model 


\section{Conclusions}

Two different moment-rotation relations for torsional mode response of an elastomeric bushing have been studied and compared. The first is the implicit but exact model of Section 4 and is defined by the equations of a boundary value problem used to simulate the exact bushing response. The second relation is the explicit but approximate model of Section 6 (PipkinRogers model) and is expressed in terms of a moment relaxation property determined from the exact model.

The moment relaxation property in the explicit moment-rotation relation was determined by a method which extrapolates results obtained from the boundary value problem representing the exact bushing response. The extrapolated and exact moment relaxation functions were in very close agreement. Moment histories corresponding to several specified rotation histories were then calculated using the exact implicit model and the approximate explicit Pipkin-Rogers model. The results were in very close agreement and indicate that the proposed explicit moment-rotation relation and the method for determining the moment relaxation function will give a very good approximation to the exact response. It thus appears to be quite suitable for use in multi-body dynamics codes.

It is useful to make several comments about the proposed moment-rotation relation. First, the present study is carried out for one specific constitutive equation. Second, comparisons were carried out for only a limited number of rotation histories. It would be useful to know constitutive properties or rotation histories which produce poorer agreement and hence provide a better test of the proposed moment-rotation relation. Finally, the results have been shown to be satisfactory over only a limited time range. This may be acceptable for use in multi-body dynamics simulations involving short time intervals such as when a tire hits a bump or a pot-hole.

\section{References}

[1] Ledesma, R., Ma, Z.-D., Hulbert, G., Wineman, A.: A nonlinear viscoelastic bushing element in multibody dynamics. Comp. Mech. 17, 287-296 (1996.)

[2] Adkins, J. E., Gent, A. N.: Load-deflexion relations of rubber bush mountings. Br. J. Appl. Phys. 5, $354-358(1954)$.

[3] Morman, K. N., Jr., Kao, B. G., Nagtegaal, J. C.: Finite element analysis of viscoelastic elastomeric structures vibrating about non-linear statically stressed configurations. Fourth International Conference on Vehicle Structural Mechanics, Society of Automotive Engineers, Detroit, MI, 83-92 (1981).

[4] Wineman, A., VanDyke, T., Shi, S.: A nonlinear viscoelastic model for one dimensional response of elastomeric bushings. Report of the Ford Durability Center, Department of Mechanical Engineering and Applied Mechanics, University of Michigan (1995).

[5] Coleman, B. D., Noll, W.: Foundations of linear viscoelasticity. Rev. Modern Phys. 33, 239-249 (1961).

[6] Lianis, G.: Constitutive equations for viscoelastic solids under finite deformation. Purdue University Report AA\&ES 63-11 (1963).

[7] Goldberg, W., Bernstein, B., Lianis, G.: The exponential extension ratio history, comparison of theory with experiment. Purdue University Report AA\&ES 67-1 (1967).

[8] McGuirt, C. W., Lianis, G.: The constant stretch rate history, comparison of theory with experiment. Purdue University Report AA\&ES 67-2 (1967).

[9] Goldberg, W., Lianis, G.: Behavior of viscoelastic media under small sinusoidal oscillations superposed on finite strain. Purdue University Report AA\&ES 67-3 (1967). 
[10] McGuirt, C. W., Lianis, G.: Constitutive equations for viscoelastic solids under finite uniaxial and biaxial deformations. Trans. Soc. Rheol. 14, 117-134 (1970).

[11] Flowers, R. J., Lianis, G.: Viscoelastic tests under finite strain and variable strain rate. Trans. Soc. Rheol. 14, 441-459 (1970).

[12] Pipkin, A. C., Rogers, T. G.: A non-linear integral representation for viscoelastic behavior. J. Mech. Phys. Solids 16, 59-72 (1968).

[13] Lee, S. B.: A study of a nonlinear viscoelastic model of elastomeric bushing response. Ph. D. Thesis, The University of Michigan, Ann Arbor (1997).

[14] Faires, J. D., Burden, R. L.: Numerical methods. 270-312. Boston: PWS 1993.

[15] Dennis, J. E., Jr.: Nonlinear least squares and equations. The state of the art in numerical analysis. (Jacobs, D., ed.) pp. 269-312. New York: Academic Press 1977.

[16] Dennis, J. E., Jr., Schnabel, R. B.: Numerical methods for unconstrained optimization and nonlinear equations, pp. 218-238. New Jersey: Prentice-Hall 1983.

Author's address: S. B. Lee and A. Wineman, Department of Mechanical Engineering and Applied Mechanics, University of Michigan, Ann Arbor, MI 48109, U.S.A. 